Shakespeare et le jeu

\title{
Avant-propos
}

\section{Pierre Kapitaniak}

\section{(2) OpenEdition \\ Journals}

\section{Édition électronique}

URL : http://journals.openedition.org/shakespeare/650

DOI : 10.4000/shakespeare.650

ISSN : 2271-6424

\section{Éditeur}

Société Française Shakespeare

\section{Édition imprimée}

Date de publication : 1 novembre 2005

Pagination : 3-5

ISBN : 2-9521475-2-3

\section{Référence électronique}

Pierre Kapitaniak, "Avant-propos », Actes des congrès de la Société française Shakespeare [En ligne], 23 | 2005, mis en ligne le 01 janvier 2007, consulté le 29 avril 2019. URL : http:// journals.openedition.org/shakespeare/650 ; DOI : 10.4000/shakespeare.650 


\section{Shakespeare et le jeu}

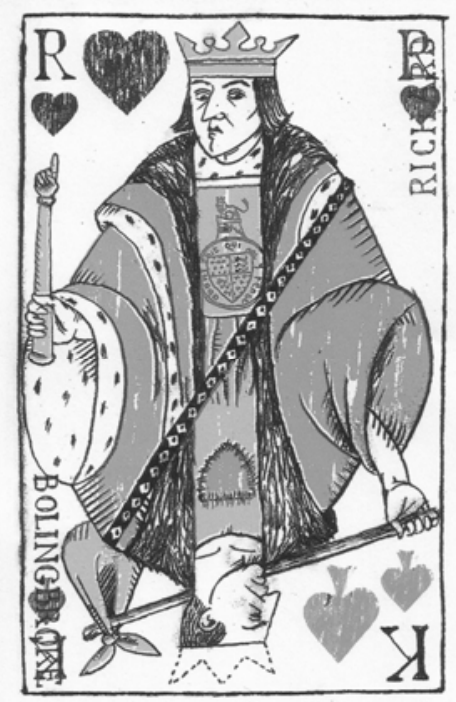

actes du Congrès organisé par la

SOCIÉTÉ FRANÇAISE SHAKESPEARE les 10, 11 et 12 mars 2005

textes réunis par Pierre KAPITANIAK sous la direction de Yves PEYRÉ 


\title{
COMITÉ SCIENTIFIQUE :
}

\author{
Margaret Jones-Davies \\ Gisèle Venet \\ Jean-Marie Maguin \\ Yves Peyré \\ François Laroque \\ Pierre Kapitaniak
}

COUVERTURE :

Edouard Lekston, King and Kusin, 2005

edlek@free.fr

conception graphique et logo

Pierre Kapitaniak

(C) 2005 Société Française Shakespeare

Institut du Monde Anglophone

Université de Paris III - Sorbonne Nouvelle

http://recherche.univ-montp3.fr/SFS/

5 rue de l'École de Médecine

75006 Paris

ISBN 2-9521475-2-3

Tous droits de traduction, de reproduction et d'adaptation réservés pour tous les pays 


\section{AVANT-PROPOS}

Tout lecteur de Shakespeare garde vivant à l'esprit la vignette montrant un jeune couple d'amoureux, Miranda et Ferdinand, jouant aux échecs sous l'œil vigilant et bienveillant de Prospero.

Johan Huizinga et, de façon plus systématique après lui, Roger Caillois ont démontré depuis longtemps à quel point le jeu, et plus particulièrement « les jeux d'imitation et d'illusion », "préfigurent les actes du spectacle ${ }^{1} »$. Lieu de représentation, de divertissement, et de jeux de toutes sortes, le théâtre est à la fois le cadre dans lequel ce jeu peut prendre place et l'aboutissement du jeu d'acteurs constitutif de l'art dramatique. Il n'est de meilleur exemple de la complexité des rapports entre jeu et théâtre que le traitement que Thomas Middleton réserve au jeu d'échecs à la fin du règne de Jacques I ${ }^{\text {er }}$ dans $A$ Game at Chess, une partie grandeur nature qui se révèle être une pièce à clefs très polémique ${ }^{2}$.

Les articles réunis dans le présent volume abordent ces relations principalement selon trois perspectives à la fois différentes et complémentaires.

La première examine le fonctionnement des jeux comme motifs dans les pièces de Shakespeare ou de ses contemporains. Guillaume Winter nous livre ainsi un panorama de motifs ludiques, allant des jeux de dés ou de cartes aux activités sportives, dans plusieurs pièces de Shakespeare. Josée Nuyts-Giornal se penche sur l'intertexte entre la gravure morale et l'exploration du motif du jeu dans King John. JeanClaude Mailhol opère une mise en perspective grâce à un travail sur plusieurs contemporains de Shakespeare au nombre desquels on compte Robert Yarington et Thomas Heywood. Enfin, Alessandro Arcangeli et Pascale Drouet nous offrent un précieux pendant contextuel en examinant respectivement deux textes exactement

\footnotetext{
${ }^{1}$ Roger Caillois, Les jeux et les hommes, Paris, Gallimard, 1967 (1958), p. 20. Cf. aussi Johan Huizinga, Homo Ludens, Londres, Temple Smith, 1970 (1938), p. 167-9.

${ }^{2}$ Voir Thomas Middleton, A Game at Chess/Une partie d'échecs (1624), texte établi, traduit, présenté et annoté par Antoine Ertlé (http://www.univ-paris3.fr/recherche/sites/ edea/iris/episteme/revue/ee_5/ee_5_art_ertle.html).
} 
contemporains, les Second Fruites de John Florio et A Notable Discovery of Cozenage de Robert Greene.

La deuxième approche privilégie le jeu linguistique, l'art du jeu de mots sous toutes ses formes, et la possibilité de voir dans celui-ci une clef de lecture du texte dramatique. C'est l'objectif de John Jowett sur la polysémie du nom du héros éponyme de Sir Thom as More ; c'est celui de Margaret Tudeau-Clayton qui analyse l'extravagance de Shakespeare dans le traitement de la langue en particulier dans The Merchant of Venice et Twelfth Night ; et c'est aussi celui de Nathalie Vienne-Guerrin qui se penche sur les «joutes de jactance» dans Henry IV.

La troisième perspective exploite la dimension histrionique de l'art dramatique en mettant en avant le jeu de l'acteur et, de façon plus moderne, celui du metteur en scène. À la fois tributaire d'un texte qui fixe des règles, l'acteur et le metteur en scène disposent d'un « jeu » ou d'une marge dans l'interprétation qu'ils peuvent en faire. Deux articles sont consacrés à des interprétations cinématographiques de pièces particulières : Sarah Hatchuel s'attache à la façon de représenter la mort dans Richard III et Russell Jackson relève les problèmes dans la transposition à l'écran d'As You Like It. Estelle Rivier et Isabelle Schwartz-Gastine, quant à elles, explorent les richesses des mises en scène shakespeariennes dans le théâtre français.

Mais cette exploration ne serait pas complète sans donner la parole aux comédiens, principaux acteurs de ce jeu scénique, ce que fait Jean-Michel Déprats en animant une rencontre avec la comédienne Sian Thomas.

Si la réflexion sur le jeu est essentielle à toute forme théâtrale, depuis la Grèce antique jusqu'au théâtre contemporain, elle acquiert à l'époque de Shakespeare un caractère supplémentaire d'actualité et d'urgence, qu'abordent les articles de Pascale Drouet et Guillaume Winter. Dans le Londres du tournant du XVI ${ }^{\mathrm{e}}$ et XVII ${ }^{\mathrm{e}}$ siècles, les acteurs des théâtres publics, sis dans des quartiers interlopes, côtoient des montreurs d'ours, des danseurs de gigues, et jouent en permanence sur ces interactions. La proximité et l'amalgame avec les lieux de plaisir, qu'il s'agisse de prostitution, de boisson ou de ces « unlawful games", 
tout autant que l'essence ludique du spectacle dramatique, attirent sur les troupes de comédiens les foudres de la censure puritaine qui se manifeste tout au long de la carrière de Shakespeare par des pressions auprès des instances législatives et une pléthore de pamphlets écrits tout autant contre le théâtre que contre les "unlawful games ", dont les champions sont John Northbrook, Stephen Gossson, Philip Stubbes et un peu plus tard William Prynne ${ }^{3}$. Dans le cas du théâtre, ils auront gain de cause en mettant fin simultanément au règne et à la vie de Charles I ${ }^{\mathrm{er}}$ ainsi qu'à toute activité théâtrale...

Enfin, pour terminer sur une note plus optimiste et purement ludique, Edouard Lekston nous propose en fin de volume de nous rapprocher davantage encore du jeu dans sa pratique, de jouer avec Shakespeare sous la forme de vignettes/rébus inspirés par ses pièces.

Pierre Kapitaniak

3 John Northbrooke, A Treatise against Dicing, Dancing, Plays, and Interludes. With other idle pastimes (1577); Stephen Gossson, Playes Confuted in fiue Actions (1582); Philip Stubbes, Of Stage-playes and Enterluds (1583); William Prynne, Histrio-Mastix (1633). 